\title{
IMAGEM CORPORAL E RISCO PARA DESENVOLVIMENTO DE TRANSTORNOS ALIMENTARES: UMA REVISÃO INTEGRATIVA DA LITERATURA
}

\author{
Body image and the risk of food disorder development: an integrative \\ literature review
}

Deisi Patrícia Volpattoㅜ; Fábia Benetti²

\footnotetext{
${ }^{1}$ Nutricionista Graduada pela Universidade Regional Integrada do Alto Uruguai e das Missões (URI) - Câmpus Frederico Westphalen.E-mail: deisipatricia2009@hotmail.com

${ }^{2}$ Nutricionista. Mestre em Envelhecimento Humano. Coordenadora e Docente do Curso de Nutrição da URI - Câmpus Frederico Westphalen.
}

Data do recebimento: 22/07/2020 - Data do aceite: 22/02/2021

\begin{abstract}
RESUMO: Transtornos alimentares ou transtornos do comportamento alimentar relacionam-se a quadros psiquiátricos da relação das pessoas para com a alimentação. Nota-se que cada vez mais, a influência da mídia, da família e da moda são capazes de influenciar o comportamento alimentar de jovens. A preocupação excessiva com o peso e a forma física em geral, principalmente em mulheres, tem acarretado no desenvolvimento de transtornos alimentares. Diante disso, este estudo objetivou analisar a associação da imagem corporal com o risco para desenvolvimento de transtornos alimentares por meio de uma revisão integrativa da literatura. Para tanto, foi realizada uma revisão bibliográfica, com consulta nas bases de dados Scientific Electronic Library Online (SciELO), PUBMED e Literatura Latino- Americana em Ciências de Saúde (LILACS), buscando-se por estudos em que se tenha utilizado o teste EAT-26, um dos instrumentos mais aplicados para o rastreamento de sintomas e comportamentos de risco para o desenvolvimento de transtornos alimentares. Identificou-se nos 16 artigos selecionados que cerca de 64\% deles associavam transtornos alimentares a imagem corporal. Com isso, observa-se que a insatisfação com a imagem corporal é uma das causas capazes de originar risco do desenvolvimento de transtornos alimentares e com isso práticas inadequadas de controle de peso.
\end{abstract}

Palavras-chave: Comportamento alimentar. Saúde. Aparência física. Alimentação. 
ABSTRACT: Eating disorders or eating behavior disorders are related to psychiatric conditions of people's relationship to food. More and more the influence of the media, family and fashion are able to influence the eating behavior of young people. Excessive concern about weight and fitness in general, especially in women, has led to the development of eating disorders. Therefore, this study aimed to analyze the association of body image with the risk of developing eating disorders through an integrative literature review. To this end, a bibliographic review was carried out, consulting the Scientific Electronic Library Online (SciELO), PUBMED and Latin American Literature in Health Sciences (LILACS) databases, looking for studies in which the eating attitudes (EAT-26) one of the most applied instruments for tracking symptoms and risk behaviors in the development of eating disorders. Among the 16 selected articles, $64 \%$ of them associated eating disorders with body image. Thus, it is observed that dissatisfaction with body image is one of the causes capable of creating a risk for the development of eating disorders and, therefore, inadequate weight control practices.

Keywords: Eating behavior. Health. Physical appearance. Food.

\section{Introdução}

Desde o início do século XX há uma supervalorização do corpo magro, musculoso e definido, que tem sido visto como sinal de poder e beleza e não de um corpo desnutrido, com sinal de pobreza ou de presença de alguma doença infecciosa como no passado (SILVA et al., 2012).

A mídia, a família e a moda são capazes de influenciar o comportamento alimentar de jovens, levando a uma preocupação excessiva e patológica com o peso, principalmente em jovens do sexo feminino. A complexidade multifatorial está relacionada a diversos distúrbios alimentares, associados a uma ideia negativa da imagem corporal, medo de engordar e por esses motivos, levam os indivíduos a aderirem a métodos inadequados para controle do peso (SILVA et al., 2012).

De acordo com o Manual Diagnóstico e Estatístico de Transtornos Mentais- DSM -V, elaborado pela Associação Psiquiátrica Americana (APA), os transtornos alimenta- res são perturbações na alimentação ou nos comportamentos pertinentes à alimentação, resultando em uma alteração no consumo dos alimentos, o que leva a um comprometimento dos indivíduos em sua saúde física e no seu funcionamento psicossocial (APA, 2014).

Ainda, de acordo com o DSM-V, dentre os transtornos alimentares, podem ser citados como principais a anorexia nervosa e a bulimia nervosa, sendo ambos os distúrbios alimentares em que há abstenção voluntária ou ingestão compulsiva de alimentos, seguida de métodos purgativos (BORGES et al., 2006).

As patologias descritas apresentam em comum uma preocupação exacerbada com o peso, medo de engordar e uma visão distorcida da forma corpórea. No caso da anorexia nervosa há uma perda intensa de peso. O paciente com anorexia utiliza-se geralmente de dietas rígidas, apresentando medo de ganhar peso e percepções distorcidas sobre o próprio corpo, muito embora na maioria dos casos o indivíduo possua um peso abaixo da média normal para sua estatura, biótipo, idade e gênero (MORAES, 2010). 
Já a bulimia nervosa refere-se a uma ingestão de alimentos de forma exacerbada, seguida de vômitos induzidos e do uso de medicamentos como diuréticos e laxantes. O paciente com bulimia apresenta uma grande preocupação com o peso e com a imagem corporal, e episódios de compulsão alimentar, que passam a ocorrer com o passar do tempo de forma mais comum, em todas as situações que podem gerar sentimentos negativos, como é o caso de momentos de solidão, de frustração, tristeza e outros mais (CORDÁS, 2004).

Os transtornos alimentares (TA) são patologias graves e com alto grau de morbidade, e tem prevalência em adolescentes do sexo feminino, sendo representado por $90 \%$ dos casos e $10 \%$ dos casos do sexo masculino. Esses transtornos têm aumentado nas últimas décadas e tomado uma proporção enorme e, por isso, vêm se tornando alvo de muitos estudos (NOBREGA, 2008).

Os TA tem relação com transtornos psiquiátricos, a frequente busca do corpo perfeito, a insatisfação com a imagem corporal, baixa autoestima que, consequentemente, podem levar a bulimia e a anorexia nervosa (SOUZA et al., 2011).

Um dos instrumentos mais utilizados para o rastreamento de sintomas e comportamento de risco de transtornos alimentares é o Eating Atitudes Test (EAT). Em sua versão com 26 itens são analisados três fatores: dieta, bulimia e preocupação com alimentos e autocontrole oral. A dieta reflete restrição dietética para alimentos com altos valores calóricos e preocupação excessiva com magreza, já a bulimia e preocupação com alimentos refere-se a episódios de ingestão exagerada de alimentos, seguidos por métodos patológicos de controle de peso corporal e autocontrole oral que representa o autocontrole em relação à comida e avalia a influência que o ambiente pode exercer sobre os hábitos alimentares (FORTES et al., 2016).
Diante disso, este estudo objetivou analisar a associação da imagem corporal com o risco para desenvolvimento de transtornos alimentares relatados pela literatura científica por meio de uma revisão integrativa da literatura.

\section{Material e Métodos}

Trata-se de uma revisão integrativa da literatura que, segundo Vosgerau e Romanowsk (2014), refere-se a um método que proporciona a síntese de conhecimento e a incorporação e aplicabilidade de resultados de estudos na prática.

Para a realização deste estudo foram consultados apenas artigos científicos indexados, nos idiomas português e inglês, com restrição de ano de publicação (a partir de 2015) contemplando, desta maneira, publicações dos últimos cinco anos. As buscas foram realizadas nos meses de abril a junho de 2020, nas seguintes bases de dados: Scientific Electronic Library Online (SciELO), PubMed e Literatura Latino-Americana em Ciências de Saúde (LILACS).

Como palavras-chave para a realização das buscas utilizou-se: "Transtornos alimentares" AND "Insatisfação corporal" AND "Teste de atitudes alimentares" e "Eating disorders", AND "Body dissatisfaction", AND "Eating attitudes test".

O descritor "teste de atitudes alimentares" ou "Eating atitudes test" não é um descritor comum, presente nos descritores de ciências da saúde (DECS) ou usualmente utilizado, entretanto, por um teste validado, internacionalmente, para avaliar risco para desenvolvimento de transtornos alimentares, achamos pertinente revisar artigos que tivessem aplicado esse teste, como meio de padronização dos resultados.

Foram considerados artigos originais indexados que contemplavam a especificidade 
do tema e estejam disponíveis na íntegra. Os critérios de exclusão utilizados foram: estudos não científicos, estudos em outras línguas que não português e inglês, estudos com animais, artigos de acesso restrito e de revisão bibliográfica.

No total foram localizados 59 artigos que, ao aplicar os critérios de exclusão restaram 16 estudos. A extração dos dados e avaliação dos estudos incluídos foram realizadas por dois revisores independentes, a partir dos títulos, seguidos por leitura dos resumos e, após, leitura do artigo na íntegra.

A limitação desta revisão inclui o pequeno número de artigos indexados encontrados, possivelmente ocasionado pela limitação das palavras-chave utilizadas como critério de inclusão.

\section{Resultados e Discussão}

No que se refere aos transtornos alimentares esta revisão propõe-se a evidenciar estudos voltados ao uso do teste EAT- 26 para a detecção de anorexia e bulimia. Neste sentido, este estudo utilizou-se da pesquisa integrada dos descritores, para identificar artigos sobre esta temática. Resultando em 16 trabalhos encontrados.

Os estudos incluidos no presente estudo são descritos no Quadro 1, sendo apontado o ano de sua publicação, autores, objetivo geral, metodologia utilizada e amostra.

Pesquisado o período dos últimos 05 (cinco) anos obtiveram-se os seguintes dados anuais quanto ao número de artigos publicados por ano, como pode ser observado na Figura 1.

Analisando os artigos selecionados, notase que no ano de 2016 realizou-se o maior número de publicações sobre a detecção de anorexia e bulimia com o teste EAT 26, sendo que no ano de 2019 foram encontrados apenas três artigos sobre a temática. Com isso, reforça-se a necessidade de que sejam realizadas novas e atuais pesquisas sobre o tema.

Figura 1 - Número de publicações anuais

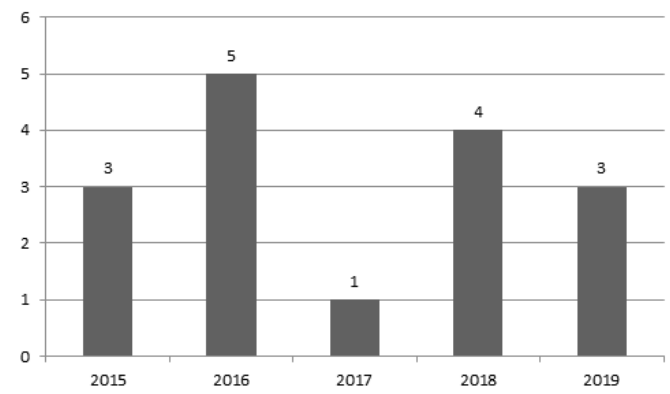

Fonte: VOLPATTO; BENETTI, 2020.

Frente aos dados trazidos no Quadro 1, percebe-se que dos 16 artigos encontrados, 9 deles associavam transtornos alimentares à imagem corporal, o que representa cerca de $64 \%$ dos artigos encontrados.

De acordo com Batista et al. (2015) há uma relação entre a insatisfação com a imagem corporal e os transtornos alimentares. Assim a insatisfação corporal pode ser compreendida como uma das causas que originam práticas inadequadas de controle de peso e o risco do desenvolvimento de transtornos alimentares.

Fortes et al. (2015) identificou em seu estudo que a insatisfação corporal e a internalização do ideal de magreza estiveram relacionadas às três sub escalas do EAT-26 (Dieta, Bulimia e Autocontrole Oral). As adolescentes com baixa autoestima estiveram mais propensas a comportamentos bulímicos; as adolescentes insatisfeitas com a imagem corporal estiveram mais sujeitas a restrições alimentares e comportamentos compulsivos e purgativos; e aquelas que mais internalizaram o ideal de magreza estiveram mais vulneráveis à dieta, bulimia e autocontrole oral. 


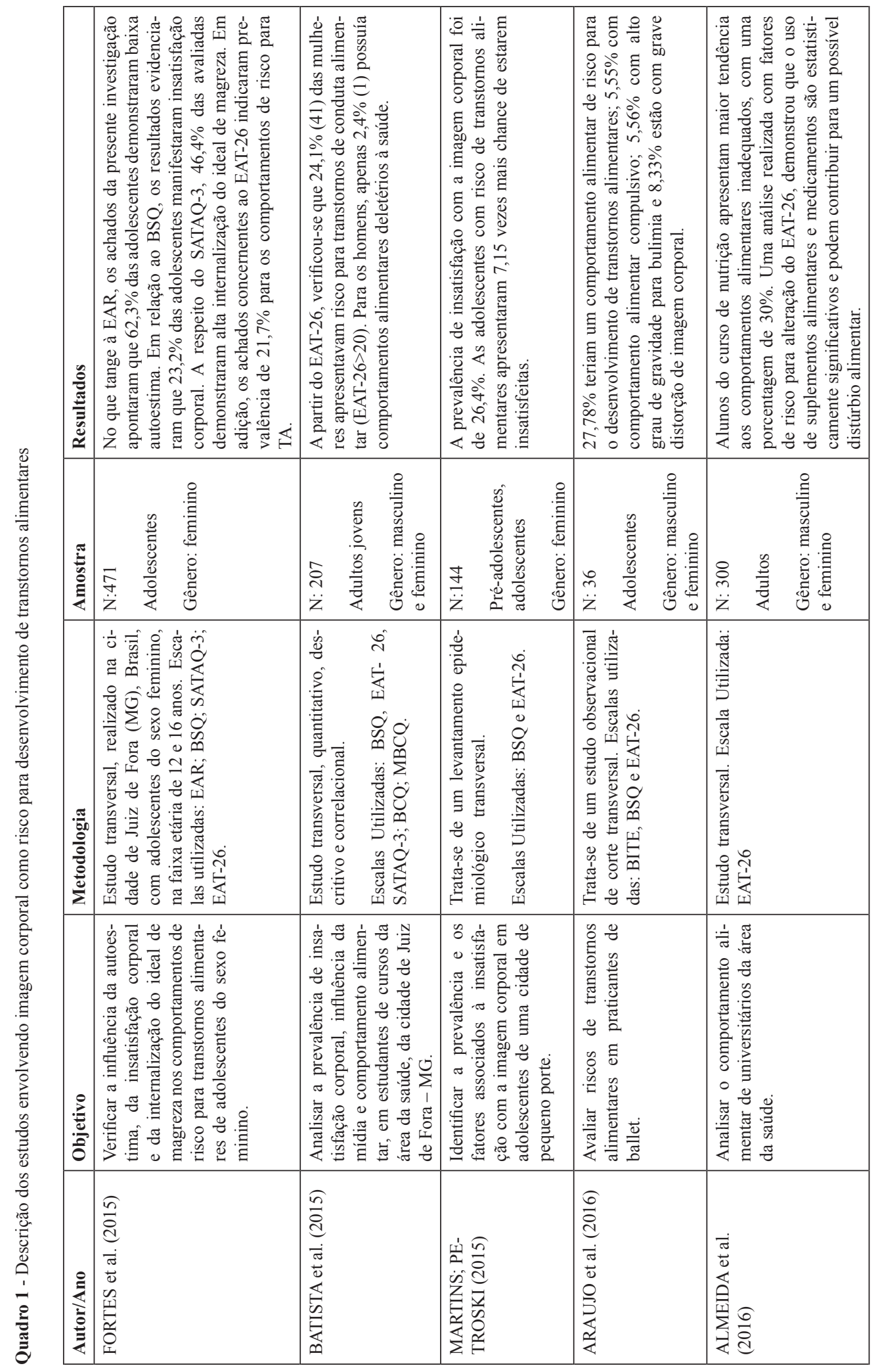




\begin{tabular}{|c|c|c|c|}
\hline 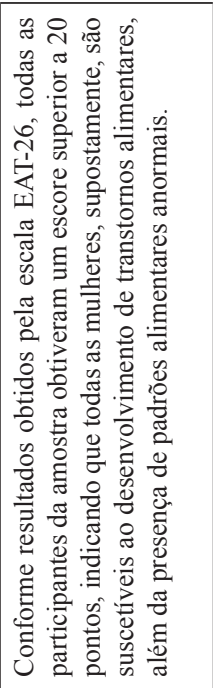 & 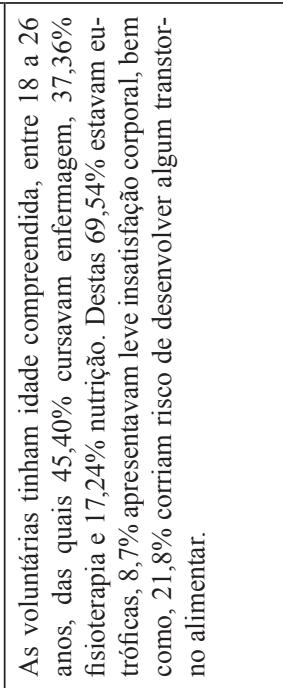 & 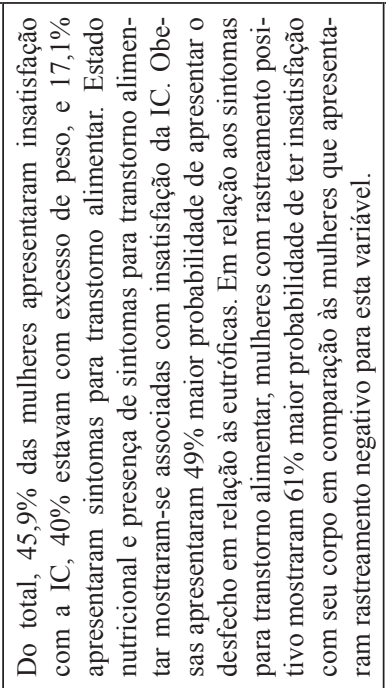 & 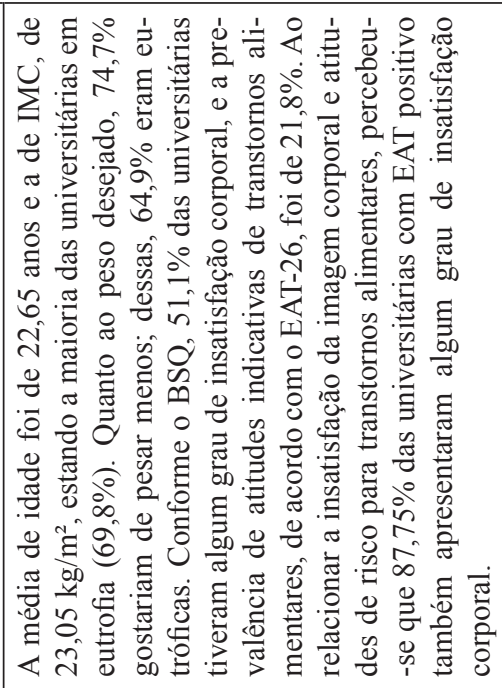 \\
\hline 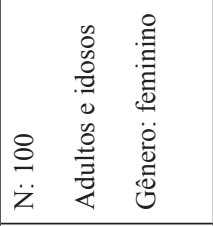 & 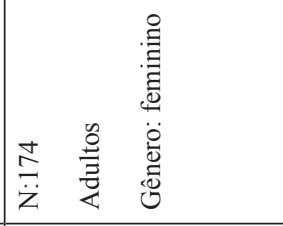 & 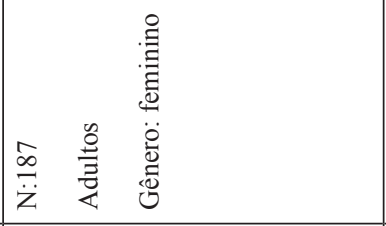 & 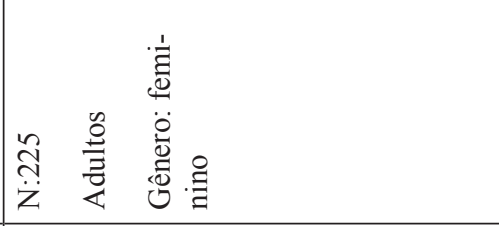 \\
\hline 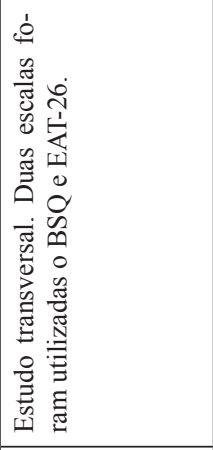 & 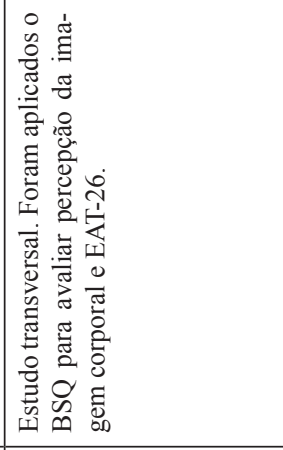 & 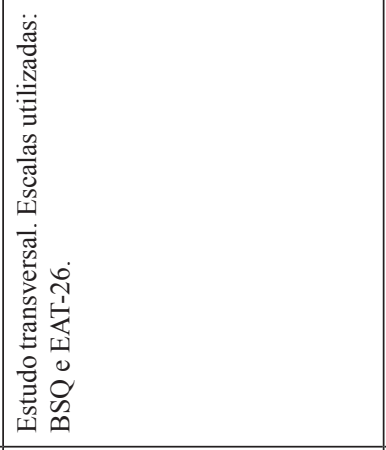 & 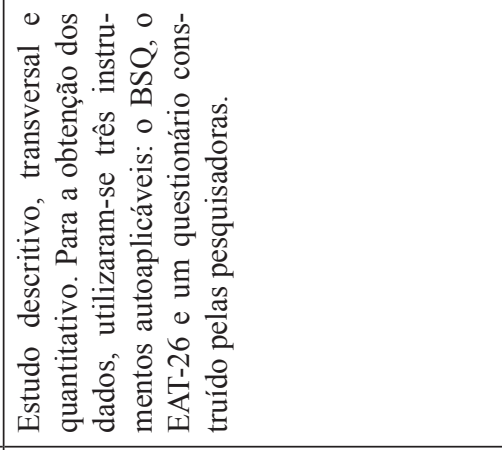 \\
\hline 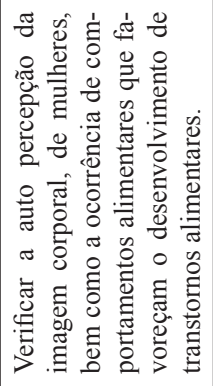 & 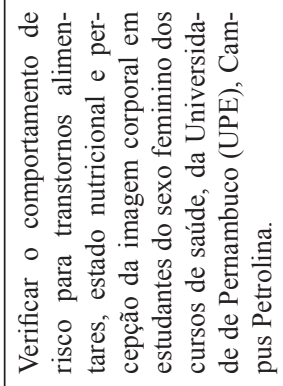 & 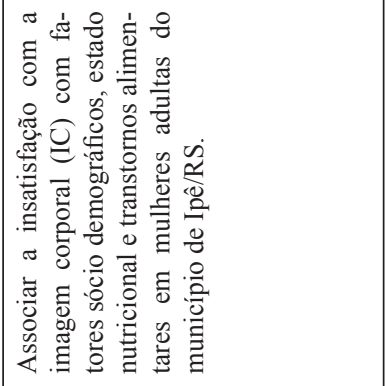 & 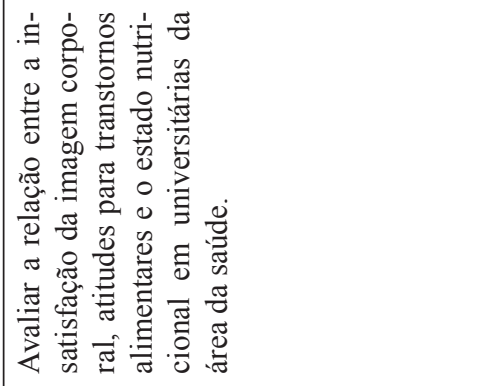 \\
\hline 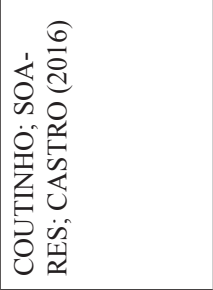 & 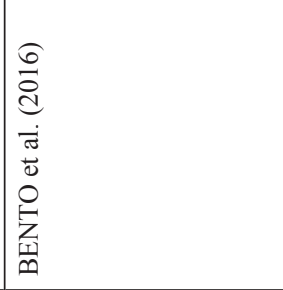 & 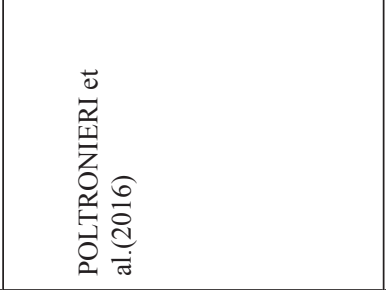 & 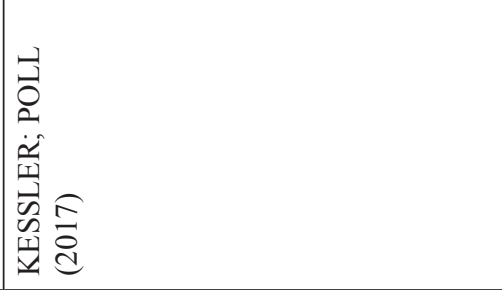 \\
\hline
\end{tabular}




\begin{tabular}{|c|c|c|c|c|}
\hline 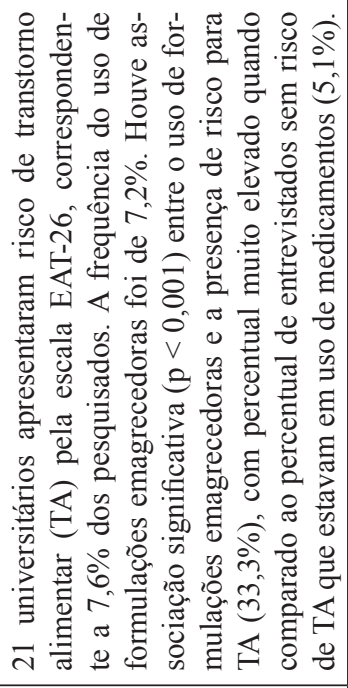 & 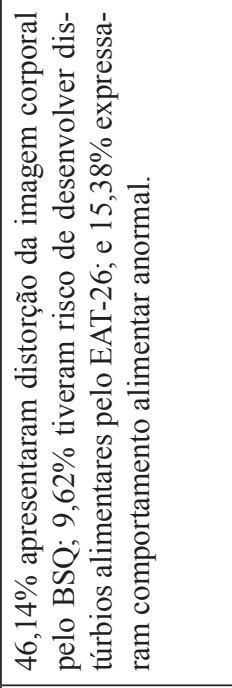 & 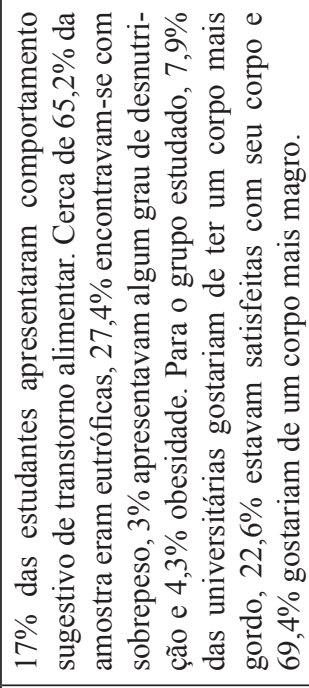 & 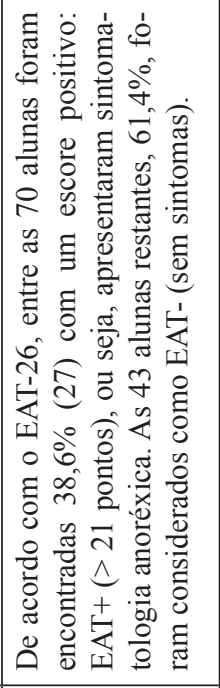 & 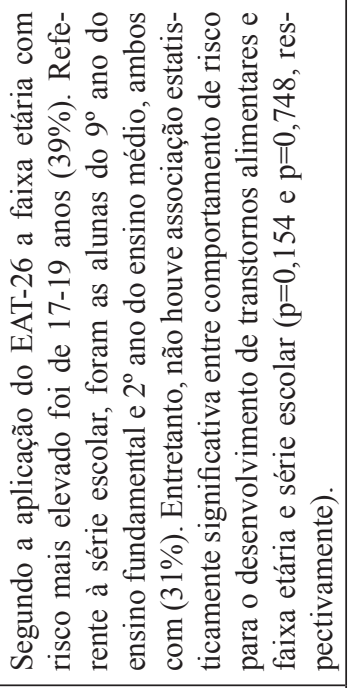 \\
\hline 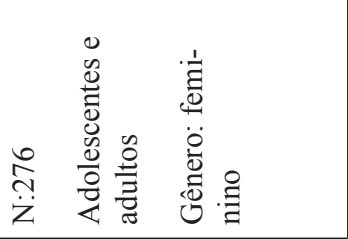 & 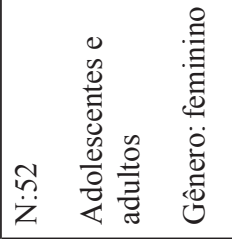 & 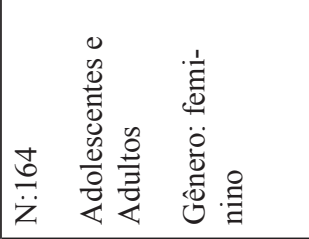 & 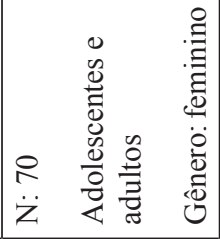 & 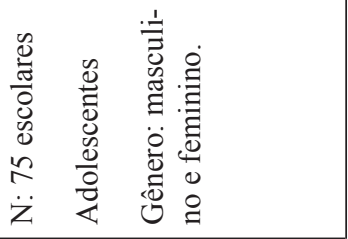 \\
\hline 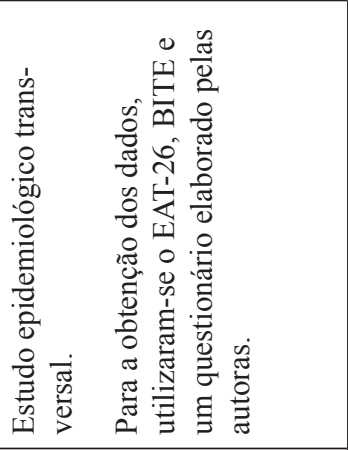 & 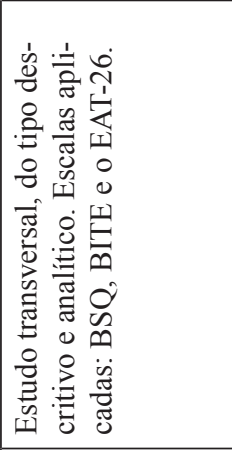 & 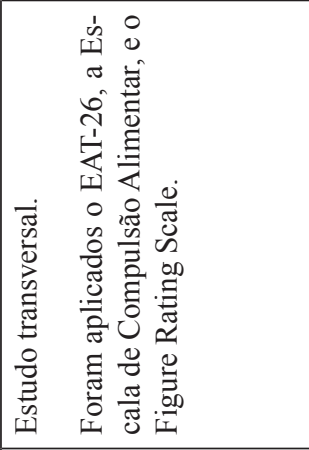 & 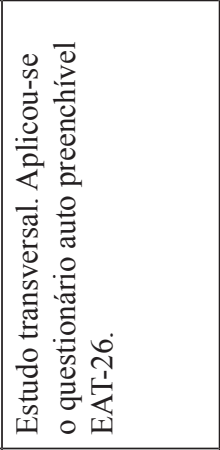 & 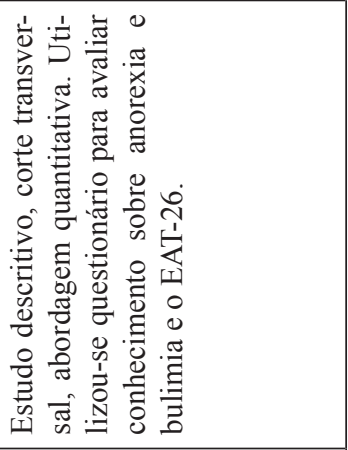 \\
\hline 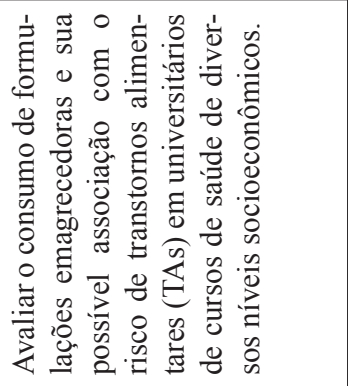 & 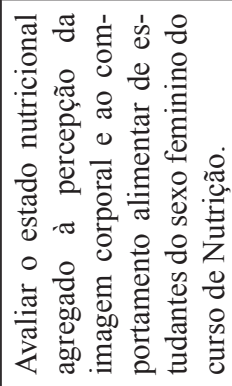 & 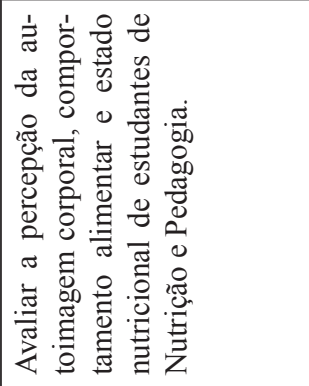 & 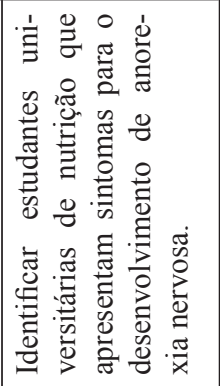 & 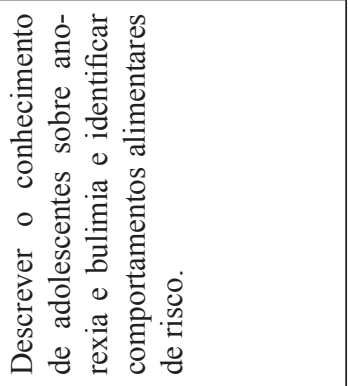 \\
\hline 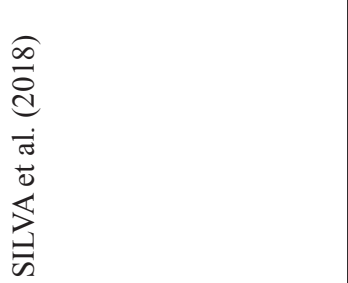 & 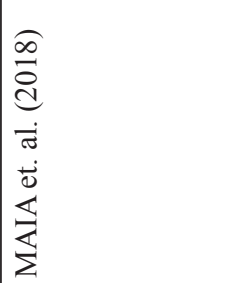 & 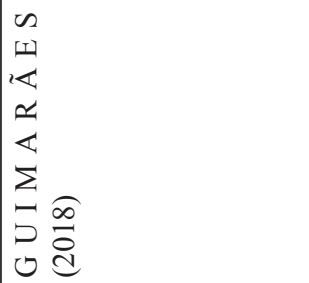 & 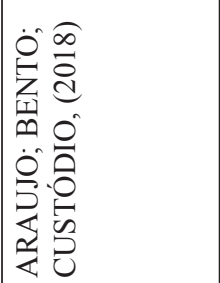 & 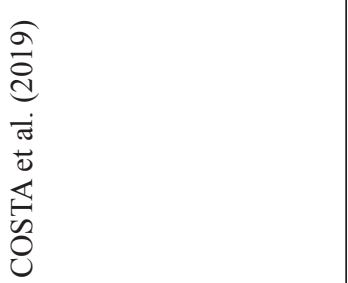 \\
\hline
\end{tabular}




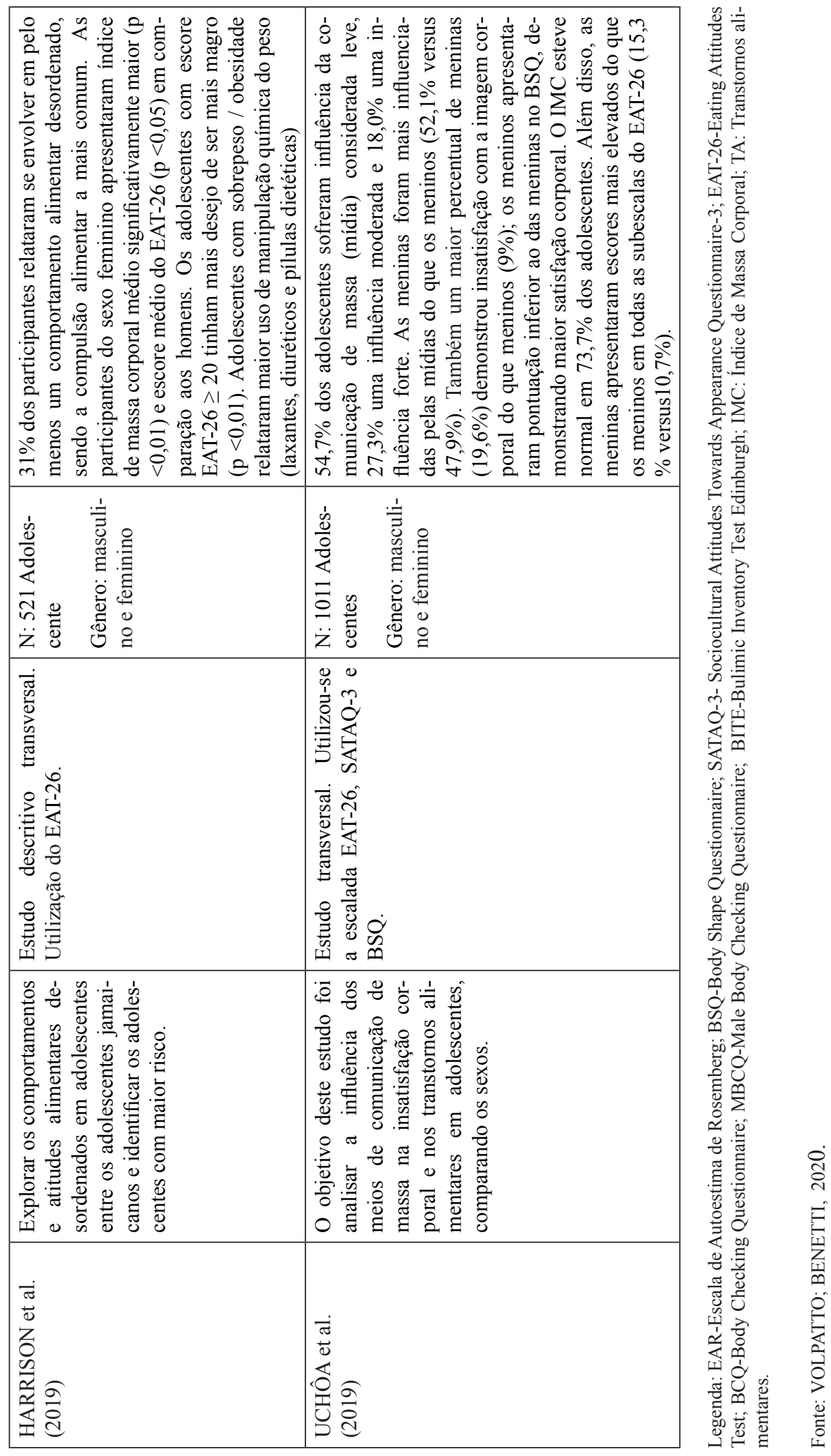


No estudo de Martins e Petroski (2015) a prevalência de insatisfação com a imagem corporal foi de $26.4 \%$. As adolescentes com risco de transtornos alimentares apresentaram 7,15 vezes mais chance de estarem insatisfeitas.

Kessler e Poll (2017) em sua pesquisa com 225 universitárias matriculadas em oito cursos da área da saúde, utilizando a escala EAT-26, ao relacionar a insatisfação da imagem corporal e atitudes de risco para transtornos alimentares, percebeu-se que $87,75 \%$ das universitárias com EAT positivo, também, apresentaram algum grau de insatisfação corporal.

Dos trabalhos estudados, 11(69\%) deles foram realizados apenas com participantes do sexo feminino e $5(31 \%)$ deles com ambos os sexos, conforme Figura 3.

As mulheres são mais suscetíveis a transtornos alimentares, dentre os fatores que levam a essa condição estão os fatores socioculturais, em uma sociedade em que há uma supervalorização do corpo feminino e a idealização da magreza (SILVA et al., 2018).

Coutinho et al. (2016) analisaram $100 \mathrm{mu}-$ lheres entre 18 a 62 anos utilizando a escala EAT-26, que mede padrões alimentares. Conforme resultados obtidos pela escala EAT-26, todas as participantes da amostra obtiveram um escore superior a 20 pontos, indicando que todas as mulheres, supostamente, foram suscetíveis ao desenvolvimento de transtornos alimentares, além da presença de padrões alimentares anormais.

Poltronieri et al. (2016) investigaram 187 mulheres entre 18 e 59 anos. Do total, 45,9\% das mulheres apresentaram insatisfação com a IC, $40 \%$ estavam com excesso de peso, e $17,1 \%$ apresentaram sintomas para transtorno alimentar. Estado nutricional e presença de sintomas para transtorno alimentar mostraram-se associados com insatisfação da IC. Participantes obesas apresentaram 49\% maior probabilidade de apresentar o desfecho em relação às eutróficas. Em relação aos sintomas para transtorno alimentar, mulheres com rastreamento positivo mostraram $61 \%$ maior probabilidade de ter insatisfação com seu corpo em comparação às mulheres que apresentaram rastreamento negativo para esta variável.

Araujo et al. (2018), em um estudo com 36 praticantes de ballet, avaliou os riscos de transtornos alimentares junto a estas mulheres. De acordo com os autores $27,78 \%$ teriam um comportamento alimentar de risco para o desenvolvimento de transtornos alimentares; $5,55 \%$ com comportamento alimentar compulsivo; $5,56 \%$ com alto grau de gravidade para bulimia e $8,33 \%$ estão com grave distorção de imagem corporal.

A presença maior de transtornos alimentares em mulheres pode ser vista no estudo de Batista et al. (2015) que em sua pesquisa investigaram 207 acadêmicos de ambos os sexos, verificando que $24,1 \%$ (41) das mulheres apresentavam risco para transtornos de conduta alimentar (EAT-26>20), já para os homens, esse número era de $2,4 \%$.

Uch et al. (2019) em estudo com 527 meninas e 484 meninos comparou os sexos ao analisar a influência dos meios de comunicação de massa na insatisfação corporal e nos transtornos alimentares em adolescentes. De acordo com os autores a influência da mídia de massa está associada a uma maior probabilidade de adolescentes apresentarem insatisfação corporal. Um aumento na insatisfação corporal está associado a um risco aumentado de desenvolver transtorno alimentar em adolescentes de ambos os sexos, mas é maior em meninas do que em meninos. Além disso, a influência do IMC na insatisfação corporal em ambos os sexos; e observando um risco de transtorno alimentar em meninas e meninos.

Nos últimos anos tem-se observado um aumento na incidência dos comportamentos 
de risco a transtornos alimentares em jovens universitárias, conforme demonstra Maia e colaboradores (2018).

Dos trabalhos analisados seis deles tinham como público alvo acadêmicas/ universitárias, representando $37 \%$ dos artigos analisados.

Almeida et al. (2016) ao analisar o comportamento alimentar de universitários da área da saúde, investigou 300 acadêmicos de ambos os sexos. De acordo com os autores, alunos do curso de nutrição apresentam maior tendência aos comportamentos alimentares inadequados, com uma porcentagem de $30 \%$.

Bento et al. (2016) analisaram 174 universitárias e identificaram que, das participantes $45,40 \%$ cursavam enfermagem; 37,36\%, fisioterapia e 17,24\%, nutrição. Destas 69,54\% estavam eutróficas, $8,7 \%$ apresentavam leve insatisfação corporal, bem como, $21,8 \%$ corriam risco de desenvolver algum transtorno alimentar.

Guimarães (2019) avaliou junto as 164 estudantes do sexo feminino a percepção da autoimagem corporal, comportamento alimentar e estado nutricional. De acordo com a autora, 17\% das estudantes apresentaram comportamento sugestivo de transtorno alimentar. Cerca de 65,2\% da amostra eram eutróficas, $27,4 \%$ encontravam-se com sobrepeso, 3\% apresentavam algum grau de desnutrição e 4,3\% obesidade. Para o grupo estudado, 7,9\% das universitárias gostariam de ter um corpo mais gordo, $22,6 \%$ estavam satisfeitas com seu corpo e $69,4 \%$ gostariam de um corpo mais magro.

Na pesquisa de Silva et al (2018) realizada com 276 universitários matriculados em quatro cursos da área da saúde, contataram que 21 universitários apresentaram risco de transtorno alimentar (TA) pela escala EAT26 , correspondente a 7,6\% dos pesquisados.

Araujo et al. (2018) aplicaram o questionário EAT-26 à 70 universitárias de nutrição, no primeiro ano da graduação, de uma instituição particular do Distrito Federal. De acordo com o EAT-26, entre as 70 alunas foram encontradas $38,6 \%$ (27) com um escore positivo: EAT + ( $>21$ pontos), ou seja, apresentaram sintomatologia anoréxica. As 43 alunas restantes, $61,4 \%$, foram considerados como EAT- (sem sintomas).

Maia et al. (2016) em estudo com 52 estudantes, do curso de Nutrição do sexo feminino, verificou que $9,62 \%$ tiveram risco de desenvolver distúrbios alimentares pelo EAT-26; e 15,38\% expressaram comportamento alimentar anormal.

Além de estudantes universitários foi possível verificar a presença de escolares adolescentes como amostra de pesquisa nos estudos analisados. An et al. (2019) exploraram os comportamentos e atitudes alimentares desordenados em 521 participantes do ensino médio (mulheres, $n=292$ ), com idades entre 11 e 19 anos. Identificaram os autores que $31 \%$ dos participantes relataram se envolver em pelo menos um comportamento alimentar desordenado, sendo a compulsão alimentar a mais comum. As participantes do sexo feminino apresentaram índice de massa corporal médio significativamente maior $(p<0,01)$ e escore médio do EAT-26 ( $\mathrm{p}<0,05)$ em comparação aos homens. Os adolescentes com escore EAT- $26 \geq 20$ tinham desejo de serem mais magros $(p<0,01)$. Adolescentes com sobrepeso / obesidade relataram maior uso de manipulação química do peso (laxantes, diuréticos e pílulas dietéticas).

Costa et al. (2019) investigaram junto a 75 escolares o conhecimento sobre anorexia e bulimia e buscou identificar comportamentos alimentares de risco. Com a aplicação do EAT-26 identificou que a faixa etária com risco mais elevado foi de 17-19 anos (39\%). Referente à série escolar, foram as alunas do $9^{\circ}$ ano do ensino fundamental e $2^{\circ}$ ano do ensino médio, ambos com (31\%). Entretanto, não houve associação, estatisticamente signi- 
ficativa, entre comportamento de risco para o desenvolvimento de transtornos alimentares e faixa etária e série escolar $(p=0,154$ e $\mathrm{p}=0,748$, respectivamente).

\section{Considerações finais}

Realizada a revisão integrativa, foi possível identificar 16 artigos atinentes a temática do desenvolvimento dos transtornos alimentares, principalmente no que se refere a distúrbios da imagem corporal. Identificou-se que a insatisfação corporal e a baixa autoestima estiveram ligadas as três sub escalas do EAT-26 (Dieta, Bulimia e Autocontrole Oral), sendo que adolescentes que apresentaram essa condição são mais propensas a comportamentos bulímicos, restrições alimentares e comportamentos compulsivos e purgativos. Ainda, de acordo com os autores analisados, as participantes do sexo feminino foram a maioria, o que ocorre, dentre outros fatores, pelo fato das mulheres serem mais suscetí- veis a transtornos alimentares, com relação aos homens.

Frente ao apresentado, nota-se a associação da insatisfação corporal com os transtornos alimentares, principalmente no que se refere a mulheres adolescentes e jovens. É importante salientar que a insatisfação com a imagem corporal sobre forte influência das mídias sociais, que cultuam historicamente um corpo magro, visto como belo e bem sucedido. Surge com isso, a necessidade de que haja uma atenção especial dos governos, da família e da sociedade como um todo, no desenvolvimento de espaços, como por exemplos as escolas, que venham a discutir essa temática, e a aceitação de todas as formas de corpos. Trata-se de um assunto de grande complexidade que exige um apoio multiprofissional, tanto na discussão do tema, como, também, no tratamento de pessoas já acometidas por transtornos alimentares, uma vez que estes trazem grande impacto para a saúde das pessoas.

\title{
REFERÊNCIAS
}

\begin{abstract}
ALMEIDA, L.C.; PIOLOGO, L. F.; BARBOSA, L. G.; OLIVEIRA NETO, J.G. Triagem de transtornos alimentares em estudantes universitários da área de saúde. Revista Brasileira de Neurologia e Psiquiatria. 2016. Disponível em: https://www.revneuropsiq.com.br/r bnp/article/ view/163. Acesso em: 31 maio 2020.
\end{abstract}

ARAUJO, M.; SILVA, A.G.; SILVA, J.J.; SILVA M.C.E. Avaliação de transtornos alimentares em praticantes de ballet. Revista Saúde, v. 10. n. 1-2. 2016. Disponível em: http://revistas.ung.br/index. $\mathrm{php} / \mathrm{saud}$ e/article/view/2106. Acesso em: 31 maio 2020.

ARAUJO, T. S.; BENTO, F. C. J. C; CUSTÓDIO, M. R. M. Risco para o desenvolvimento de anorexia nervosa em estudantes universitárias de nutrição. Revista Cientifica Sena Aires. 2018. Disponível em: http://revistafacesa.senaaires.com.br/index.php/revisa/ article/view/320/230. Acesso em: 20 maio 2020.

BATISTA, A. et al. Dimensão atitudinal da imagem corporal e comportamento alimentar em graduandos de educação física, nutrição e estética da cidade de Juiz De Fora - MG. Revista de educação física, v. 26, n. 1, 2015. Disponível em: http://www.scielo.br/scielo.php?script=sci arttext\&pid=S198330832015000100069\&lng=en\&nrm=iso. Acesso em: 25 maio 2020. 
BENTO, K. B M.; ANDRADE, K. N. D. S.; SILVA, E.I.G.; MENDES, M. L. M.; OMENA, C. M. B.; CARVALHO, P. G. S.; SCHWINGEL, P. A. Transtornos alimentares, imagem corporal e estado nutricional em universitárias de Petrolina-PE. Revista Brasileira de Ciências da Saúde, v. 20, n. 3, p.197-202, 2016. Disponível em: https://periodicos.ufpb.br/ojs/index.php/rbcs/article/view/26418. Acesso em: 12 maio 2020.

BRANDT, L. M. T. Comportamento de risco para bulimia em adolescentes. Revista Paulista de Pediatria, v. 37, n. 2. p.217-224, 2019. Disponível em: http://www.scielo.br/pdf/rpp/v37n2/pt_01030582-rpp-2019-37-2-00008.pdf. Acesso em: 05 out. 2019.

CORDAS, T. A.; CLAUDINO, A. M. Transtornos alimentares: fundamentos históricos.Revista Brasileira de Psiquiatria, v. 24, n. 3, p. 3-6, 2002.

COSTA, J. R. S.; ALMEIDA, P. C.; ROSCOCHE, K. G. C.; MARIANO, M. R. Conhecimento e comportamento de risco para anorexia e bulimia em adolescentes. Revista baiana de enfermagem, v. 33, n. 29, 2019. Disponível em: https://portalseer.ufba.br/index.php/em fermagem/article/ view/29399/20180. Acesso em: 31 maio 2020.

COUTINHO, É. J.; SOARES, M. J.; CASTRO, N. R. Relação entre a imagem corporal e os comportamentos alimentares com os transtornos alimentares anorexia nervosa e bulimia nervosa.

Revista Científica Univiçosa, v. 8, n. 1, p. 816-822, 2016.

DSM-V 2013. Manual de Diagnósticos e Estatística da Associação Norte-Americana de

Psiquiatria. Psiquiatria Geral. Disponível em: http://www.psiquiatriager al.com.br/dsm4/sub_index. htm. Acesso em: 05 out. 2019.

FORTES, L.S. Autoestima, insatisfação corporal e internalização do ideal de magreza influenciam os comportamentos de risco para transtornos alimentares? Revista de Nutrição, v. 28, n. 3, 2015. Disponível em: https://www.scielo.br/scielo.php?script $=$ sci_arttext\&p id=S141552732015000300253. Acesso em: 21 maio 2020.

GUIMARÃES, I. C. T. Estado nutricional, avaliação de transtornos alimentares e autoimagem corporal em universitárias do rio de janeiro. Revista Brasileira de Obesidade, Nutrição e Emagrecimento, v.12. n. 70, p.196-204, 2018. Disponível em: http://www.rbone.com.br/index.php/ rbone/article/view/677. Acesso em: 31 maio 2020.

HARRISON, A.N.; BATEMAN, J. C. C. B.; YOUNGER-COLEMAN, N. O. M.; WILLIAMS, M.C.; ROCKE, K.D.; CLATO-DAY S, S. C.; CHANG, S. M. Disordered eating behaviours and attitudes among adolescentes in a middle-income country. Eating and Weight Disorders - Studies on Anorexia, Bulimia and Obesity, v. 25, p.1727-1737, 2020.

KESSLER, A. L.; POLL, F. A. Relação entre imagem corporal, atitudes para transtornos alimentares e estado nutricional em universitárias da área da saúde. Jornal brasileiro de psiquiatria, $v$. 67, n. 2, p. 118-125. June 2018. Disponível em: http://www.scielo.br/scielo.php?script=sci arttext\&pid=S0047-20852018000200118\&lng=en\&nrm=iso. Acesso em: 01 maio 2020.

MAIA, R. G. L. Estado nutricional e transtorno de comportamento alimentar em estudantes do curso de graduação em nutrição do instituto federal de educação, ciência e tecnologia, Ceará Brasil. Revista Demetra, v. 1, n. 13, p. 135-145, 2018.

MARTINS, C. R.; PETROSKI, E. L. Insatisfação com a imagem corporal em adolescentes do sexo feminino de uma cidade de pequeno porte: prevalência e correlações. Motricidade, v. 11, n. 2, p. 94106, jun. 2015. Disponível em: http://www.scielo.mec.pt/scielo.php?script=sci_arttext\&pid=S1646107X2015000200010\&lng=pt\&nrm=iso. Acesso em: 20 Abr. 2020.

NÓBREGA, F. J. O que você quer saber sobre Nutrição: Perguntas e respostas comentadas - 1 . Ed. São Paulo: Editora Manole, 2008. 
POLTRONIERIA, T. S.; TUSSET, C.; GREGOLETTO, M. L. O.; CREMONESE, C. Insatisfação com a imagem corporal e fatores associados em mulheres do sul do Brasil. Revista Ciência \& Saúde Coletiva, v. 9. n. 3. 2016. Disponível em: http://revistaseletronicas.pucrs.br/ojs/index.php/ faenfi/article/view/21770. Acesso em: 31 maio 2020.

SILVA, J. D.; SILVA, A. B. J.; OLIVEIRA, K. V.A; NEMER, A.S.A. Influência do estado nutricional no risco para transtornos alimentares em estudantes de nutrição. Ciência e Saúde Coletiva, v. 17, n. 12, p. 3399-3406, 2012.

SILVA, G. A. Consumption of dietary substances and their association with risk of eating disorders in college students. Jornal Brasileiro de Psiquiatria, v. 67, n. 4, p. 239-246, 2018 . Disponível em: http://www.scielo.br/scielo.php ?script=sci_arttext\&pid=S0047-20852018000400239\&lng=en\& nrm=iso. Acesso em: 31 maio 2020.

UCHÔA, F. N. M.; UCHÔA, N.M.; DANIELE, T.M.D.C; LUSTOSA, R.P.; GARRIDO, N. D.; DEANA, N. F.; ARANHA, Á. C. M.; ALVES, N. Influence of the Mass Media and Body Dissatisfaction on the Risk in Adolescents of Developing Eating Disorders. International Journal of Environmental Research and Public Health, v. 16, n. 9, 1508, 2019. Disponível em: https:// www.mdpi.com/1660-4601/16/9/1508. Acesso em: 01 Abr. 2020.

VOSGERAU, D. S. A. R.; ROMANOWSKI, J. P. Estudos de revisão: implicações conceituais e metodológicas. Diálogo Educacional, v. 14, n. 41, p. 165-189, jan./abr. 2014.

SOUZA, A. A. Estudo sobre a anorexia e bulimia nervosa em universitárias. Psicologia: Teoria e Pesquisa, v. 27, n. 2, p. 195-198, 2011. Disponível em: https://periodicos.unb.br/index.php/ revistaptp/article/view/17523. Acesso em: 25 Abr. 2020. 
